\title{
Evaluation of efficacy and safety of epalrestat and epalrestat in combination with methylcobalamin in patients with diabetic neuropathy in a randomized, comparative trial
}

\author{
Manish Maladkar ${ }^{1^{*}}$, N. J. S. Saggu ${ }^{2}$, P. Moralwar ${ }^{3}$, Aziz A. Mhate $^{4}$, Datta Zemse ${ }^{5}$, Anil Bhoraskar ${ }^{6}$ \\ ${ }^{1}$ 23-A Shah Industrial Estate, Andheri, India; Corresponding Author: scientific@aristopharma.org \\ ${ }^{2}$ M.G.M. Hospital, Mumbai, India \\ ${ }^{3}$ Moralwar Day Care Centre, Sector 1-A, Mumbai, India \\ ${ }^{4}$ Mhate Clinic, B-3, Sector 2, Mumbai, India \\ ${ }^{5}$ F-1, Dattaguru Co-Operative Society, Mumbai, India \\ ${ }^{6}$ Bhalchandra Niwas, Mumbai, India
}

Received 7 November 2012; revised 9 December 2012; accepted 18 December 2012

\section{ABSTRACT}

Recent Background: Diabetic neuropathy is one of the major complications in long standing hyperglycemic patients. Though exact mechanism of neuronal damage is unclear, accumulation of excess sorbitol through polyol pathway is believed to contribute significantly. Epalrestat and methylcobalamin are extensively used in this area to counter neuronal damage. This study was aimed to evaluate the combined effect of these drugs. Materials and Methods: A total of 220 patients with diabetic neuropathy were included in this study. The patients were divided into two groups; group A was administered combination of epalrestat $50 \mathrm{mg}$ and methylcobalamin $500 \mathrm{mcg}$ while group B was administered epalrestat $50 \mathrm{mg}$ alone (both thrice daily). The treatment period was 12 weeks with monitoring on week 4, 8 and 12 of the study. At baseline and at follow up visits following parameters were evaluated: loss of sensation, burning sensation, numbness, muscle cramps, spontaneous pain, weakness, dizziness, loss of the thermal sensitivity, tendon reflexes, muscle strength and pain intensity using visual analog scale (VAS). Results: All the parameters were improved in both the groups compared to baseline. In group A significant improvement was seen on week 4 itself and continued for the rest of the study in all the measured parameters. Group B showed significant improvement from 8th week onwards. The inter-group difference is statistically significant in favour of the combination therapy. Conclu- sion: Combination of epalrestat and methylcobalamin is a better option for the treatment of diabetic neuropathy than epalrestat alone. Combination therapy was associated with faster onset and better symptomatic relief.

Keywords: Epalrestat; Methylcobalamin;

Diabetic Neuropathy

\section{INTRODUCTION}

Diabetes is a multifaceted disease associated with neurological, vascular, immunological and metabolic complications. Long standing hyperglycemia is believed to be the major cause of these complications. Diabetic neuropathy is a common complication that develops in about $50 \%$ of people with diabetes [1]. The prevalence of up to $7 \%$ is reported in South Indian patients even at the time of diagnosis of diabetes [2]. Diabetic neuropathy has widespread occurrence and devastating effects.

The precise pathogenesis of diabetic neuropathy is unclear despite recent advances. Polyol pathway of glucose metabolism has been considered as one of the major mechanisms in the pathogenesis of diabetic neuropathy $[2,3]$. Conversion of glucose to sorbitol by the enzyme aldose reductase is the rate limiting step of polyol pathway. Increased activity of polyol pathway due to hyperglycemia and subsequent accumulation of excess sorbitol explains the neuronal damage in diabetes [4].

Epalrestat is a carboxylic acid derivative that acts as aldose reductase inhibitor. Epalrestat is proven to have beneficial effects in diabetic neuropathy in many controlled clinical trials. In hyperglycemia, epalrestat significantly reduces intracellular sorbitol accumulation by 
an uncompetitive aldose reductase inhibition. Epalrestat improves motor and sensory nerve conduction velocity and subjective neuropathy symptoms in patients with diabetic neuropathy [4-7].

Methylcobalamin is one of the biologically active forms of vitamin B12. It is used in the treatment of peripheral neuropathy, diabetic neuropathy, and as a preliminary treatment for amyotrophic lateral sclerosis. Unlike cyanocobalamin, methylcobalamin is active in the spinal fluid. Due to this property, it is able to help heal the damaged nerve cells and restores normal functions. In clinical studies, methylcobalamin showed improvement in the somatic and autonomic symptoms with regression of signs of diabetic neuropathy such as pain and paresthesia [8,9].

In our previous work we demonstrated the superiority of epalrestat over methylcobalamin in the treatment of patients with diabetic neuropathy [10]. The combination of epalrestat and methylcobalamin may serve synergistically to relieve the symptoms of diabetic neuropathy. Epalrestat reduces the neuronal damage caused by sorbitol accumulation while methylcobalamin helps in rejuvenation of neurons. The objective of the present study was to compare the efficacy, safety and tolerability of epalrestat alone and in combination with methylcobalamin in patients with diabetic neuropathy.

\section{MATERIALS AND METHODS}

This phase III, single blind, randomized, comparative clinical study was conducted at 5 different centers by the qualified investigators as per ICH guidelines. Ethics committee approval was obtained prior to the study and patient recruitment was done as per inclusion-exclusion criteria after getting the informed consent. All patients were asked to do daily 20 - 30 minutes moderate exercise which includes warm up, stretch, brisk walking, swimming, gardening, cycling and jogging. Also study population was asked to abstain from alcohol consumption and smoking during the study period. Patients were instructed to follow common diet plan. The detailed study plan is given in Figure 1. The treatment period was 12 weeks and both the medications were administered thrice daily. This was a single blind study; therefore patients were unaware of treatment given to them. Additionally the investigational product was supplied in opaque containers. Patients were monitored on week 4, 8 and 12 of the study. Patient's demographic data and medical history were recorded at screening visit. Physical examination was recorded at screening and week 12 follow up visit. Systemic examination and vital examination were recorded at every follow up visit. Efficacy parameters like loss of sensation, burning sensation, numbness, muscle cramps, spontaneous pain, weakness, dizziness,

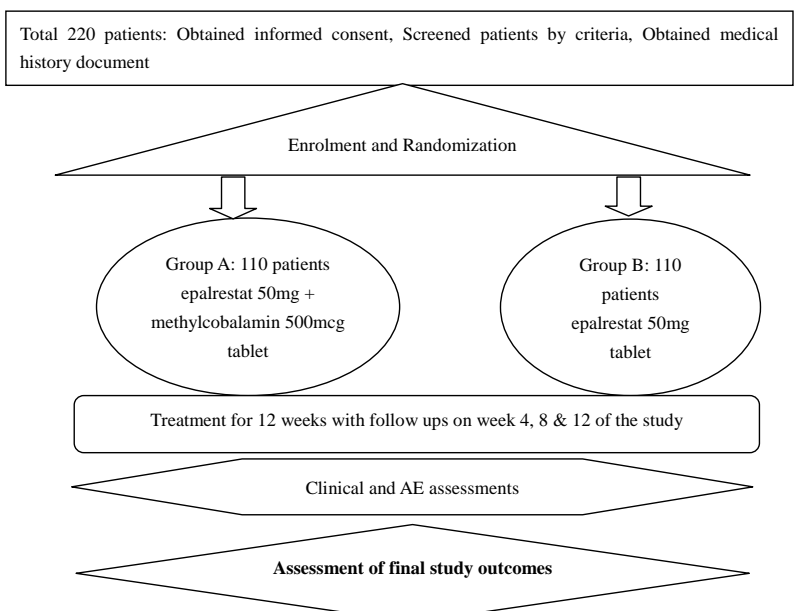

Figure 1. Study outline.

loss of sensation of heat and cold, tendon reflexes, muscle strength and pain intensity (VAS) were recorded at baseline and all follow up visits. The group of muscles evaluated for muscle strength included dorsiflexors, plantar flexors, interdigital muscles, everters, inverters and grip muscles. Laboratory tests like C.B.C., HbA1c, fasting blood sugar (FBS) and post prandial blood sugar (PPBS), LFT (Liver Function Tests) and RFT (Renal Function Tests) were recorded at baseline and at the end of treatment.

\section{Statistical Analysis}

Basic statistical evaluation including Mean, Median, $\mathrm{SD}$, etc. were calculated for the raw data. Efficacy variables such as diabetic neuropathy symptoms, tendon reflex, pain intensity and muscle strength were calculated by using Chi square test and ANOVA test.

\section{RESULTS}

Out of 220 patients with diabetic neuropathy enrolled in the study, 209 patients completed the study and were taken for final analysis. A total number of 11 patients dropped out from the study. Six patients from epalrestat + methylcobalamin combination group (group A) and five patients from epalrestat group (group B) were considered as dropouts as they failed to follow up. All the dropouts were because of inability of patients to attend the visits and not related to either efficacy or safety of the drug. The demographic profiles were not statistically different in both the groups as tabulated in Table 1. Blood glucose profiles like FBS, PPBS and HbA1c were measured before and after study. These values are tabulated in Table 2. All the vital signs were measured at the start and at the end of the study; these parameters did not reveal any significant changes during the study period. 


\subsection{Effect on Neuropathy Symptoms}

Patients in group A showed statistically significant ( $p$ $<0.05$ ) improvement in diabetic neuropathy symptoms such as loss of sensation, burning sensation, numbness, muscle cramps, spontaneous pain, weakness, dizziness and loss of thermal sensitivity from week 4 of treatment over the baseline score as shown in Table 3. Similarly patients receiving epalrestat alone showed statistically significant $(p<0.05)$ improvement in neuropathy symptoms from week 8 of treatment over the baseline.

The onset of significant relief was faster in group A (from week 4 onwards) compared to group B (week 8 onwards). At the end of week 12, both the treatments showed improvement in neuropathic symptoms. Average number of patients reporting relief in all the neuropathic symptoms in group A were higher than in group B and the difference was statistically significant $(p<0.05)$.

\subsection{Effect on Tendon Reflex Symptom Score}

Both the groups showed statistically significant $(p<$ 0.05 ) improvement over baseline tendon reflexes symptom scores. Statistical significant improvement in tendon reflex symptom score was observed at the end of week 4 of treatment in group A and at end of week 8 in group B. Number of patients reporting normal tendon reflexes

Table 1. Summary table of demographic data.

\begin{tabular}{cccc}
\hline \multicolumn{2}{c}{ Parameter } & Group A & Group B \\
\hline \multicolumn{2}{c}{ Total No. of Patients } & 110 & 110 \\
Gender & Male & $79(71.82)$ & $77(70.00)$ \\
No. (\%) & Female & $31(28.18)$ & $33(30.00)$ \\
& Mean & 50.81 & 51.00 \\
Age & Median & 50.00 & 50.00 \\
(Years) & SD & 10.76 & 05.64 \\
& Range & $25-80$ & $25-80$ \\
\hline
\end{tabular}

Table 2. Key parameters before and after the study.

\begin{tabular}{ccccc}
\hline & \multicolumn{2}{c}{ Group A } & \multicolumn{2}{c}{ Group B } \\
\cline { 2 - 5 } Parameter & $\begin{array}{c}\text { Before } \\
\text { Treatment } \\
\text { (Mean) }\end{array}$ & $\begin{array}{c}\text { After } \\
\text { Treatment } \\
\text { (Mean) }\end{array}$ & $\begin{array}{c}\text { Before } \\
\text { Treatment } \\
\text { (Mean) }\end{array}$ & $\begin{array}{c}\text { After } \\
\text { Treatment } \\
\text { (Mean) }\end{array}$ \\
FBS (mg/dl) & 170.94 & 169.74 & 179.82 & 177.89 \\
PPBS (mg/dl) & 272.15 & 267.80 & 281.80 & 279.06 \\
HbA1c (\%) & 8.18 & 8.10 & 8.21 & 8.16 \\
AST (IU/L) & 34.05 & 34.12 & 35.83 & 34.68 \\
ALT (IU/L) & 27.26 & 26.47 & 28.69 & 27.29 \\
Bilirubin & 0.70 & 0.72 & 0.70 & 0.70 \\
Total (mg/dl) & & & & \\
\hline
\end{tabular}

Table 3. Effect of both the treatments on neuropathic symptoms.

\begin{tabular}{|c|c|c|c|c|c|}
\hline \multirow{2}{*}{\multicolumn{2}{|c|}{ Evaluated Parameter }} & \multicolumn{4}{|c|}{ No. of Patients Reporting the Problem } \\
\hline & & \multirow{2}{*}{$\begin{array}{c}\text { Baseline } \\
99\end{array}$} & \multirow{2}{*}{$\frac{\text { Week } 4}{52^{* \#}}$} & \multirow{2}{*}{$\frac{\text { Week } 8}{24^{* \#}}$} & \multirow{2}{*}{$\frac{\text { Week } 12}{07^{* \#}}$} \\
\hline Loss of & Group A & & & & \\
\hline Sensation & Group B & 100 & 92 & $51^{*}$ & $27^{*}$ \\
\hline \multirow{2}{*}{$\begin{array}{l}\text { Burning } \\
\text { Sensation }\end{array}$} & Group A & 103 & $56^{*}$ & $22^{* \#}$ & $06^{*}$ \\
\hline & Group B & 101 & 78 & $43^{*}$ & $12^{*}$ \\
\hline \multirow{2}{*}{ Numbness } & Group A & 101 & $53^{*}$ & $24^{* \#}$ & $07^{*}$ \\
\hline & Group B & 102 & 81 & $47^{*}$ & $14^{*}$ \\
\hline \multirow{2}{*}{ Muscle Cramps } & Group A & 100 & $49^{*}$ & $19^{* \#}$ & $05^{* \#}$ \\
\hline & Group B & 102 & 76 & $44^{*}$ & $26^{*}$ \\
\hline \multirow{2}{*}{$\begin{array}{l}\text { Spontaneous } \\
\text { Pain }\end{array}$} & Group A & 102 & $66^{*}$ & $21^{* \#}$ & $11^{* \#}$ \\
\hline & Group B & 103 & 78 & $45^{*}$ & $28^{*}$ \\
\hline \multirow{2}{*}{ Weakness } & Group A & 86 & $44^{*}$ & $17^{* \#}$ & $06^{* \#}$ \\
\hline & Group B & 94 & 76 & $38^{*}$ & $19^{*}$ \\
\hline \multirow{2}{*}{ Dizziness } & Group A & 83 & $47^{*}$ & $15^{* \#}$ & $07^{*}$ \\
\hline & Group B & 85 & 70 & $36^{*}$ & $16^{*}$ \\
\hline \multirow{2}{*}{$\begin{array}{l}\text { Thermal } \\
\text { Sensitivity }\end{array}$} & Group A & 101 & $66^{*}$ & $28^{*}$ & $09^{*}$ \\
\hline & Group B & 102 & 86 & $46^{*}$ & $20^{*}$ \\
\hline
\end{tabular}

By Chi-Square test ${ }^{*} p<0.05$ over baseline, ${ }^{*} p<0.05$ between groups.

increased from 13 at baseline to 77 at week 12 in group A. When two groups were compared, group A showed statistically significant $(p<0.05)$ improvement in number of patients with normal tendon reflex (Table 4).

\subsection{Effect on Pain Intensity Score}

Both the groups showed reduction in mean pain intensity score from week 4 onwards which was statistically significant $(p<0.05)$ over the baseline as shown in Figure 2. Mean pain score decreased from 5.69 (severe pain) at baseline to 1.50 (mild pain) at the end of treatment in group A compared to 5.92 at baseline to 2.46 at the end of treatment in group B. Moreover, difference in mean pain intensity score between the two groups was statistically significant $(p<0.05)$ in favour of group A.

\begin{tabular}{cc}
\hline Score & Symptom \\
\hline 0 & No Pain \\
$1-2$ & Mild Pain \\
$3-4$ & Moderate Pain \\
$5-6$ & Severe Pain \\
$7-8$ & Very Severe Pain \\
$8-10$ & Worst pain \\
\hline
\end{tabular}


Table 4. Effect on tendon reflex symptom score.

\begin{tabular}{|c|c|c|c|c|c|c|}
\hline \multirow{3}{*}{$\begin{array}{l}\text { Duration } \\
\text { in Weeks }\end{array}$} & \multicolumn{6}{|c|}{ Tendon Reflexes (n = No. of Patients) } \\
\hline & \multicolumn{3}{|c|}{ Group A (n = 104) } & \multicolumn{3}{|c|}{ Group B $(\mathrm{n}=105)$} \\
\hline & Absent n (\%) & $\begin{array}{c}\text { Present with } \\
\text { Reinforcement n (\%) } \\
\end{array}$ & Normal n (\%) & $\begin{array}{l}\text { Absent } \\
\mathrm{n}(\%)\end{array}$ & $\begin{array}{c}\text { Present with } \\
\text { Reinforcement n (\%) }\end{array}$ & $\begin{array}{c}\text { Normal } \\
\mathrm{n}(\%)\end{array}$ \\
\hline Baseline & $25(24.04)$ & $66(63.46)$ & $13(12.50)$ & $22(20.95)$ & $64(60.95)$ & $19(18.10)$ \\
\hline Week 4 & $13(12.50)$ & $56(53.85)$ & *35 (33.65) & $16(15.24)$ & 59 (56.19) & $30(28.57)$ \\
\hline Week 8 & 07 (06.73) & 34 (32.69) & $\#{ }^{* *} 63(60.58)$ & $13(12.38)$ & $52(49.52)$ & *40 (38.10) \\
\hline Week 12 & 05 (04.81) & 22 (21.15) & \#*77 (74.04) & 08 (7.62) & 46 (43.81) & *51 (48.57) \\
\hline
\end{tabular}

${ }^{*} p<0.05$ over baseline, ${ }^{*} p<0.05$ between groups.

\subsection{Effect on Muscle Strength}

From week 8 onwards, both the groups showed statistically significant $(p<0.05)$ improvement in mean muscle strength score over the baseline as shown in Figure 3. The score improved from 2.58 to 3.68 during the treatment in group A compared to rise from 2.43 to 3.16 in group B. When mean score of two groups was compared at the end of week 8 and 12, the improvement in group A was more and was statistically significant ( $p<$ 0.05 ) than group B.

\subsection{Global Evaluation of Treatment}

Investigators were asked to grade the treatments as very good, good, satisfactory and unsatisfactory. Overall response suggests that investigators were of the opinion that combination of epalrestat and methylcobalamin is more efficacious than epalrestat alone (Figure 4).

\subsection{Safety Assessment}

In group A adverse events were observed in 10.91\% patients compared to $9.09 \%$ in group B. All adverse events reported were mild to moderate in nature and did not require any additional treatment, or discontinuation of the trial therapy. The difference in percentage of patients reporting adverse events in both groups was statistically insignificant.

Pathological investigations, CBC, LFT, RFT, fasting and PP blood glucose, HbA1c before and after the study did not demonstrate any statistically significant change. The measured values of vital signs such as body temperature, pulse rate, respiratory rate, systolic and diastolic blood pressure were within the normal range in all patients.

\section{DISCUSSION}

Diabetic neuropathy develops in majority of poorly controlled diabetic patients as a late complication of diabetes. Treatment is of utmost importance as $60 \%-70 \%$ of this population may progress to suffer from serious life-threatening complications. Adequate glycemic con-

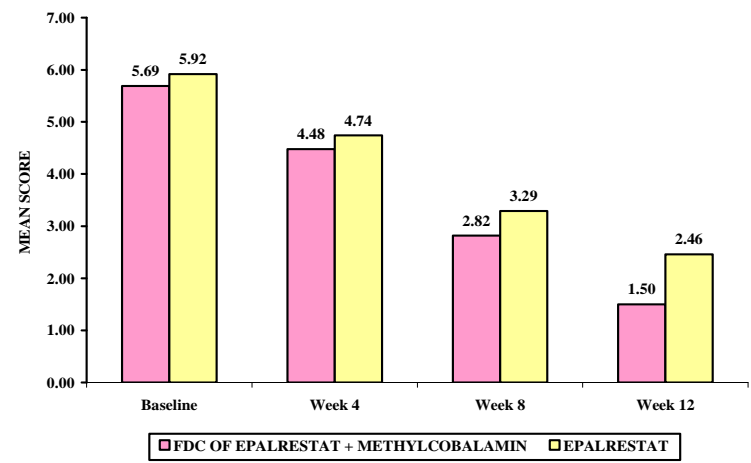

Figure 2. Pain intensity scores.

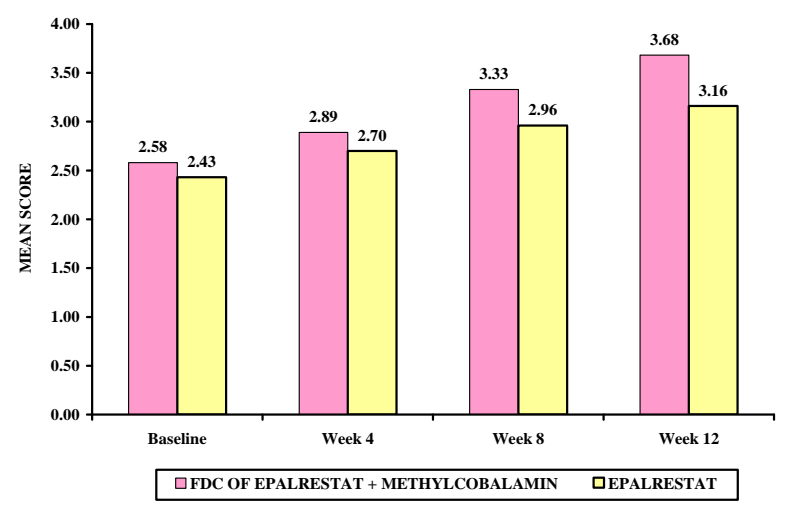

Figure 3. Muscle strength scores: 0) No muscle movement; 1) Visible muscle movement, but no movement at the joint; 2) Movement at the joint, but not against gravity; 3) Movement against gravity, but not against added resistance; 4) Movement against resistance, but less than normal; 5) Normal strength.

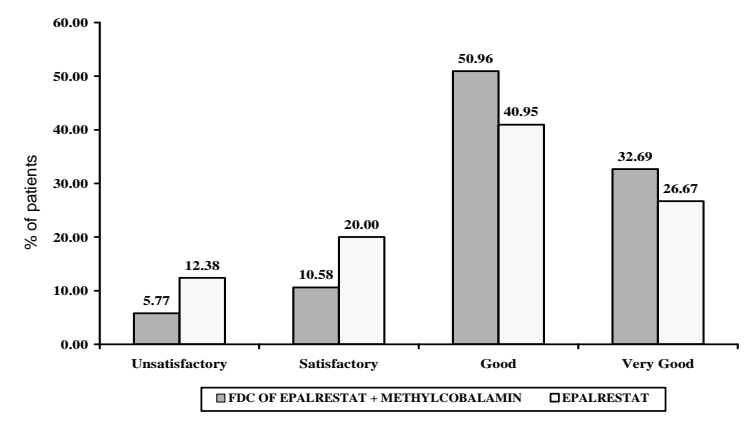

Figure 4. Global evaluation of therapy by the investigators. 
trol is the corner stone of the diabetic neuropathy treatment; however, over a period of time glycemic control declines leading to increased risk of complications. Symptomatic relief of neuropathic complaints plays a very important role in improving the quality of life of a patient with diabetic neuropathy. Anticonvulsants, tricyclic antidepressants, topical analgesics, etc. have been effectively employed in the management of neuropathic symptoms. Methylcobalamin has a long history as a nerve and it has been used in the treatment of neuropathy for a long time. Epalrestat is a relatively newer addition in this category that has gained the acceptance of the medical community as an effective treatment option for diabetic neuropathy because epalrestat may interfere with the pathogenesis of diabetic neuropathy, potentially preventing or ameliorating long-term diabetic complications [6,11].

Present study was conducted to assess the efficacy and safety of epalrestat and epalrestat in combination with methylcobalamin in treatment of patients with diabetic neuropathy. We have evaluated most common diabetic neuropathy complaints including loss of sensation, numbness, weakness, pain, loss of thermal sensitivity, dizziness, burning sensation and muscle cramps in this study.

The results of the present study reveal that combination group as well as epalrestat monotherapy showed improvement in diabetic neuropathy symptoms over the baseline. All the evaluated neuropathy symptoms showed statistically significant $(p<0.05)$ improvement in both the groups. However, in combination group symptomatic relief was achieved much earlier and was better compared to epalrestat alone.

Improvement in diabetic neuropathic patients was also investigated in terms of tendon reflex symptom score, pain intensity score, and muscle strength score. Intra group comparison of the results showed that percentage of patients with normal tendon reflex increased significantly $(p<0.05)$ from week 4 onwards in combination group and from week 8 onwards in epalrestat group.

The synergistic effect of epalrestat and methylcobalamin may be linked to their complementary mechanisms of neuroprotection. Epalrestat helps to prevent neuronal degeneration by reducing the accumulation of toxic sorbitol and decreasing the oxidative stress while methylcobalamin helps to recover neuronal injury.

The safety of both the drugs was assessed based on the incidence of adverse events reported by the patients who received the trial therapy. Previous experience also confirms the safety. Both the treatments were well tolerated and addition of methylcobalamin to epalrestat does not pose any safety concern.

\section{CONCLUSION}

The addition of methylcobalamin to epalrestat therapy is a better option in the treatment of diabetic neuropathy. Present study concludes that combination of epalrestat and methylcobalamin provides faster and better resolution of symptoms compared to epalrestat alone in patients with diabetic neuropathy.

\section{REFERENCES}

[1] Peeraer, E., Van Lutsenborg, A., Verheyen, A., De Jongh, R., Nuydens, R. and Meert, T.F. (2011) Pharmacological evaluation of rat dorsal root ganglion neurons as an in vitro model for diabetic neuropathy. Journal of Pain Research, 4, 55-65. doi:10.2147/JPR.S15452

[2] Ramachandran, A., Snehalatha, C., Vijay, V. and Vishwanathan, M. (1996) Diabetic retinopathy at the time of diagnosis of NIDDM in South Indian subjects. Diabetes Research and Clinical Practice, 32, 111-114. doi:10.1016/0168-8227(96)01185-0

[3] Chihiro, Y.N. (1998) Aldose reductase in glucose toxicity: A potential target for the prevention of diabetic complications. Pharmacological Reviews, 50, 21-33.

[4] Itagaki, I., Shimizu, K., Kamanaka, Y., Ebata, K., Kikkawa, R., Haneda, M. and Shigeta, Y. (1994) The effect of an aldose reductase inhibitor (epalrestat) on diabetic nephropathy in rats. Diabetes Research and Clinical Practice, 25, 147-154. doi:10.1016/0168-8227(94)90002-7

[5] Hotta, N., Kakuta, H., Koh, N., Fukasawa, H., Yasuma, T., Awaya, S. and Sakamoto, N. (1991) In vitro retinal and erythrocyte polyol pathway regulation by hormones and an aldose reductase inhibitor. Diabetes Research and Clinical Practice, 14, 29-35. doi:10.1016/0168-8227(91)90050-N

[6] Ramirez, M.A. and Borja, N.L. (2008) Epalrestat: An aldose reductase inhibitor for the treatment of diabetic neuropathy. Pharmacotherapy, 28, 646-655. doi:10.1592/phco.28.5.646

[7] Sharma, S.R. and Sharma, N. (2008) Epalrestat, an aldose reductase inhibitor, in diabetic neuropathy: An Indian perspective. Annals of Indian Academy Neurology, 11, 231235. doi:10.4103/0972-2327.44558

[8] Yaqub, B.A., Siddique, A. and Sulimani, R. (1992) Effects of methylcobalamin on diabetic neuropathy. Clinical Neurology and Neurosurgery, 94, 105-111. doi:10.1016/0303-8467(92)90066-C

[9] Sun, Y., Lai, M.S. and Lu, C.J. (2005) Effectiveness of vitamin B12 on diabetic neuropathy: Systematic review of clinical controlled trials. Acta Neurologica Taiwanica, 14, 48-54.

[10] Maladkar, M., Rajadhyaksha, G., Venkatswamy, N., Hariharan, R.S. and Lohati, S.R. (2009) Efficacy, safety and tolerability of epalrestat compared to methylcobalamin in patients with diabetic neuropathy. International Journal of Diabetes in Developing Countries, 29, 28-34. doi:10.4103/0973-3930.50712 